\title{
HYDRAULIC SYSTEMS ANALYSIS
}

An Introduction 


\title{
HYDRAULIC SYSTEMS ANALYSIS AN INTRODUCTION
}

\author{
JOHN STRINGER \\ Senior Lecturer, \\ Department of Mechanical Engineering, \\ University of Sheffield
}

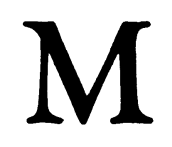


(C) J. D. Stringer, 1976

Softcover reprint of the hardcover 1st edition 1976

All rights reserved. No part of this book may be reproduced or transmitted in any form or by any means without permission.

This book is sold subject to the standard conditions of the Net Book Agreement.

First published in 1976 by

THE MACMILLAN PRESS LTD

London and Basingstoke

Associated companies in New York Dublin

Melbourne Johannesburg and Madras

SBN 333182936

ISBN 978-1-349-02599-2

DOI 10.1007/978-1-349-02597-8

ISBN 978-1-349-02597-8 (eBook) 


\section{Preface}

Engineering design calculations are usually based on approximations and simplifying assumptions rather than being exact and precise so as to ensure that results are obtained quickly and economically. Prerequisites of suitable calculation procedures are that they give results of sufficient accuracy for their purpose but are in no danger of being misleading. In the case of hydraulic systems and servomechanisms, one such technique for carrying out design calculations is based on the method of linear analysis. With this method the characteristics of the various components of any device are represented in the form of linear equations which are then taken together in order to predict how a completed system will behave. The actual behaviour of the practical device may be somewhat different from that predicted but the differences should be only in detail and not in essence.

This book traces the constituent features involved in applying linear methods to hydraulic mechanisms. A step-by-step approach is adopted with no attempt made to convert the text into a reference book. Involved are certain aspects of the subjects of dynamics and of fluid mechanics combined with the basic concepts of linear control theory and a few electrical ideas (for coping with electrohydraulics).

The text concentrates on this so-called 'small perturbation' or 'small excursion' or linearised analysis whose use in hydraulics has been developed in the past by various research workers. One pioneer in this field, under whose aegis the author has now worked for some years, is Professor J. K. Royle. More advanced techniques of analysis have also H. E. Merritt evolved and one book Hydraulic Control Systems by H. E. Merritt, dealing with both linear and other methods, is particularly recommended for further reading.

The author wishes to thank Mr K. Morris and Mr D. Puttergill for their help with diagrams, Mr K. H. Sutherland for his useful suggestions and Mrs Ivy Ashton for doing the typing.

J. D. Stringer

Sheffield, 1976 


\section{Contents}

Preface

Nomenclature $\quad$ xi

1 Fluid flow calculations 1

1.1 Power 1

1.1.1 Pumping 2

1.1.2 Pressures 2

1.2 The steady flow equation 3

1.2.1 Useful forms of the equation 4

1.2.2 Orifices 4

1.2.3 Oil temperature rise 6

1.3 Flowrates 6

$\begin{array}{ll}\text { 1.3.1 Flow through tubes } & 7\end{array}$

1.3.2 Capillary (small-bore) tubes $\quad 8$

1.3.3 Connecting pipelines $\quad 8$

1.3.4 Turbulent flow 9

1.3.5 Approximations used in calculation 9

$\begin{array}{lr}\text { 1.3.6 Annular passages } & 10\end{array}$

1.3.7 Viscosity variations 11

1.4 Compressibility 11

1.4.1 Compressibility flowrates 11

$\begin{array}{ll}\text { 1.4.2 Dilation of containers } & 12\end{array}$

$\begin{array}{ll}\text { 1.4.3 Air content } & 13\end{array}$

1.4.4 Flexible hoses $\quad 14$

$\begin{array}{ll}1.4 .5 \text { Surges } & 14\end{array}$

1.4.6 Pressure waves 16

$\begin{array}{ll}\text { Problems } & 17\end{array}$

2 Dynamic analysis 21

2.1 First-order systems 21

2.1.1 A first-order fluid system 22

2.1.2 A first-order electrical system 23

2.1.3 A first-order hydraulic servomechanism 24

2.1.4 The first-order equation $\quad 25$

2.2 The step input 26

2.2.1 Response of first-order systems to step input 27

2.2.2 Response as a function of time 27

2.3 Ramp input and response for first-order systems 28 
2.4 Harmonic input $\quad 29$

2.4.1 Harmonic response of first-order systems 29

2.4.2 Graphical representations $\quad 30$

2.4.3 Harmonic response locus $\quad 32$

$\begin{array}{ll}2.4 .4 \text { Logarithmic plots } & 33\end{array}$

$\begin{array}{lll}2.5 & \text { Second-order systems } & 34\end{array}$

2.5.1 A second-order electrical circuit $\quad 36$

$\begin{array}{ll}\text { 2.5.2 The second-order equation } & 37\end{array}$

2.6 Response of second-order systems to ramp input 39

2.7 Harmonic response of second-order systems 39

2.7.1 Harmonic response locus $\quad 40$

2.7.2 Logarithmic plots $\quad 40$

Problems 41

3 Hydraulic frequency $\quad 45$

3.1 A single-acting hydraulic jack $\quad 45$

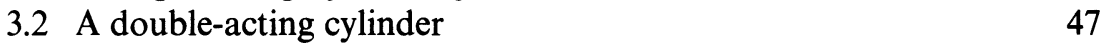

3.3 A double-acting cylinder with a long exhaust pipeline 48

3.4 An oil hydraulic motor with two pipelines $\quad 50$

Problems $\quad 52$

4 Variable pump systems $\quad 54$

4.1 The pump $\quad 55$

4.2 The motor $\quad 55$

4.3 Open loop systems $\quad 56$

4.3.1 Steady state operation $\quad 56$

$\begin{array}{ll}\text { 4.3.2 Dynamic analysis } & 57\end{array}$

4.4 Closed loop (position control) systems 58

$\begin{array}{ll}4.5 \text { Practical systems } & 60\end{array}$

$\begin{array}{lr}\text { Problem } & 60\end{array}$

5 Linear control theory $\quad 61$

5.1 Algebraic stability criterion (Routh-Hurwitz) 61

$\begin{array}{lll}5.2 & \text { Open loop relations } & 63\end{array}$

$\begin{array}{ll}\text { 5.2.1 First-order example } & 63\end{array}$

$\begin{array}{ll}\text { 5.2.2 Third-order example } & 63\end{array}$

$\begin{array}{ll}5.2 .3 \text { General case } & 65\end{array}$

5.2.4 Harmonic input $\quad 65$

5.2.5 Open loop harmonic response locus $\quad 65$

5.2.6 Open loop testing $\quad 66$

$\begin{array}{lll}5.3 & \text { Nyquist stability criterion } & 67\end{array}$

$\begin{array}{ll}5.4 \text { Adequate stability } & 67\end{array}$

$\begin{array}{ll}\text { 5.4.1 Gain and phase margins } & 67\end{array}$ 
5.4.3 Maximum closed loop dynamic magnification 69

6 Pumps

6.1 Types of pump 72

6.2 Flow irregularities 75

6.3 Constant-pressure sources 79

7 Flow through valves 81

7.1 Four-way spool valves $\quad 81$

$\begin{array}{ll}\text { 7.1.1 Critical centre valves } & 82\end{array}$

7.1.2 Flowrate prediction $\quad 83$

7.1.3 Open centre type (underlapped four-way valve) 86

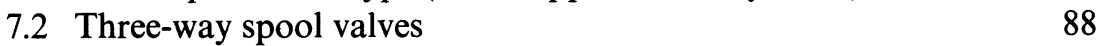

7.3 Nozzle-flapper valves $\quad 88$

Problems $\quad 90$

8 Valve-controlled systems 91

8.1 Four-way valve system 91

8.2 Pure inertia analysis $\quad 92$

8.2.1 Analysis with friction and leakage $\quad 93$

8.3 Valve position servos 95

8.3.1 The 'velocity constant' 96

8.3.2 Governing equation $\quad 96$

8.4 Feedback lever system (with a four-way valve) 98

8.5 Valve servo characteristics 99

$\begin{array}{lr}\text { 8.5.1 Stability } & 99\end{array}$

8.5.2 Harmonic response $\quad 100$

8.5.3 Description of harmonic response 101

8.5.4 Open loop characteristics 106

8.5.5 Adequate stability 106

9 Electrohydraulic servo valves 107

9.1 Flow control valves 107

9.2 Valves with coil armatures 108

9.2.1 A single-stage valve 108

9.2.2 A two-stage valve 110

9.3 Valves with torque motors $\quad 112$

9.4 Valve dynamics 114

9.4.1 Torque motors 114

9.4.2 Two-stage operation $\quad 115$

9.4.3 Simplified representations of valve characteristics $\quad 116$ 
9.5 Comments on electrical supplies 117

$\begin{array}{ll}\text { 9.5.1 Pulse width modulation } & 118\end{array}$

10 Electrohydraulic servomechanisms $\quad 120$

10.1 'Proportional' systems 121

10.1.1 Analysis 123

10.1.2 Precision $\quad 125$

$\begin{array}{ll}10.2 \text { Velocity control } & 126\end{array}$

10.2.1 Pump control 126

10.3 Compensated control 127

$\begin{array}{ll}\text { 10.3.1 Analysis } & 127\end{array}$

10.3.2 A possible electrical network 128

10.3.3 Systems with a compensating network 131

10.4 Valve characteristics 132

11 Conclusion 133

$\begin{array}{lll}\text { Appendix A Spool valve stroking forces } & 134\end{array}$

A.1 Flow forces 134

A.1.1 Steady flow 134

A.1.2 Transient flow 136

A.2 Effective moving mass 137

A.3 Frictional forces 138

A.4 Summary 139

Problem 140

Appendix B Three-way valve systems 141

B.1 Valves 141

B.1.1 Critical centre type 142

B.1.2 Open centre type (underlapped three-way valve) 142

B.2 Three-way valve system 143

B.3 Three-way valve servo 145

B.3.1 Governing equation $\quad 145$

$\begin{array}{lll}\text { Appendix C Special purpose valves } & 148\end{array}$

C.1 Poppet valves 148

C.2 Single-stage relief valve 149

C.3 A flow control valve 151

C.3.1 Forces 152

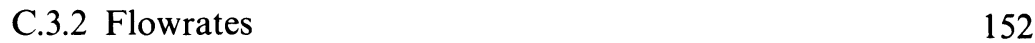

Appendix D Numerical examples 155

D.1 Question (i) 155 
D.1.1 Calculation of answers to question (i) 155

D.1.2 Answers to question (i) 159

$\begin{array}{lr}\text { D.2 Question (ii) } & 159\end{array}$

D.2.1 Calculation of answer to question (ii) 159

$\begin{array}{ll}\text { D.2.2 Answer to question (ii) } & 160\end{array}$

$\begin{array}{lll}\text { Appendix E Hydraulic lock } & 161\end{array}$

$\begin{array}{ll}\text { General Problems } & 165\end{array}$

$\begin{array}{lr}\text { References } & 169\end{array}$

$\begin{array}{lr}\text { Index } & 171\end{array}$ 


\section{Nomenclature}

a

A

$B$ c

$c_{\mathrm{r}}$

C

$C_{1}, C_{2}$

$C_{\mathrm{d}}$

$C_{\mathrm{h}}$

$d$

D

e

E

$f$

F

$g$

$g_{0}$

G

h

i

I

$I_{\mathrm{c}}$

J

$k$

K

$K_{q}$

$K_{\mathrm{c}}$

$L$

m

$M$

$n$

$N$

$N_{\mathrm{c}}$

$p$

$P$

$q$

$q$

$Q$

$r$

cross-sectional area of pipe (also a coefficient)

net area of piston

magnetic flux density

radial clearance

capacitance

constants

discharge coefficient

specific heat

diameter

$\equiv \mathrm{d} / \mathrm{d} t$

electrical potential

Young's modulus

fluid friction factor

force

gravity acceleration

force/mass conversion factor

an acceleration

a coefficient

$=(-1)^{1 / 2}$

electrical current

electrical control current

moment of inertia

a constant

a constant

valve flow coefficient

valve pressure-flow coefficient

leakage coefficient

a mass

a mass

angular speed ( $\mathrm{rad} / \mathrm{s})$

a magnitude

number of turns

complex operator

pressure or pressure difference (with various suffixes)

volume flowrate

rate of change of $q$

heat transfer rate or quantity of heat

radius 


\begin{tabular}{|c|c|}
\hline xiv & Nomenclature \\
\hline$R$ & electrical resistance \\
\hline$(R e)$ & Reynolds' number \\
\hline$s$ & complex operator \\
\hline$t$ & time \\
\hline$T$ & time constant \\
\hline$u$ & valve underlap \\
\hline$u_{1}, u_{2}$ & specific internal energies \\
\hline$v$ & velocity \\
\hline$v_{\mathrm{s}}$ & sonic velocity \\
\hline$\dot{s}$ & volume \\
\hline$W_{x}$ & external work \\
\hline$x^{n}$ & a displacement \\
\hline$y$ & a displacement \\
\hline$z$ & height above some datum \\
\hline$f$ & viscous friction rate \\
\hline $\mathscr{G}$ & a gear ratio \\
\hline $\mathscr{g}$ & Joule's equivalent \\
\hline$\ell$ & length \\
\hline $\mathscr{L}$ & inductance \\
\hline $\mathscr{M}$ & amplitude ratio \\
\hline$\imath$ & a ratio \\
\hline$t$ & temperature \\
\hline$\alpha$ & a ratio \\
\hline$\alpha_{\mathrm{p}}$ & pump capacity \\
\hline$\beta$ & bulk modulus \\
\hline$\gamma$ & angular deflection \\
\hline$\delta_{\mathrm{m}}$ & motor capacity \\
\hline$\Delta$ & a change or difference of \\
\hline$\varepsilon$ & eccentricity \\
\hline$\zeta$ & damping ratio or factor \\
\hline$\theta$ & a quantity with various suffixes \\
\hline$\lambda$ & $\begin{array}{l}\left(\theta_{\mathrm{i}} \text { input, } \theta_{\mathrm{o}} \text { output, } \theta \text { error) }\right. \\
\text { see equation C. } 2\end{array}$ \\
\hline$\mu$ & dynamic viscosity \\
\hline$v$ & kinematic viscosity (also Poisson's ratio) \\
\hline$\rho$ & density \\
\hline$\sigma$ & $=1 / \beta$ \\
\hline$\tau$ & torque \\
\hline$\phi$ & phase angle \\
\hline$\psi$ & poppet half angle \\
\hline$\omega$ & a frequency $(\mathrm{rad} / \mathrm{s})$ \\
\hline$\Omega$ & a constant velocity or rate of change \\
\hline
\end{tabular}

